\title{
Reconfiguration of 5G Radio Interface for Positioning and Communication
}

\author{
Jani Saloranta and Giuseppe Destino \\ Centre for Wireless Communications, \\ University of Oulu, Finland \\ Email: \{jani.saloranta, giuseppe.destino\}@oulu.fi
}

\begin{abstract}
In addition to high data rate, millimeter-wave technology has great potential to provide extremely high localization accuracy. In this paper, we outline the benefits of this technology for positioning and their main applications, which are no longer confined to services only but also to improve communication. We shall focus on the trade-off between data communication and positioning looking the reconfiguration mechanisms of the radio interface. Specifically, in this paper we investigate a tradeoff between achievable data rate and positioning capability via position and rotation error bound analysis, with the aim of achieving an optimal trade-off.
\end{abstract}

\section{INTRODUCTION}

One of the key innovations of $5 \mathrm{G}$ communication, the next generation of mobile networks, is considered to be millimeter wave $(\mathrm{mmW})$. Many research studies as well as 5G prototypes [1] are looking into this new technology in order to understand and verify its real rendering capabilities. [2]

In contrast to the predecessor Long Term Evolution (LTE) technology, the 5G has to address a large variety of application requirements spanning from extremely high data rate and accurate positioning capabilities to ultra-reliable communications as well as very low-latency. For this reason the development of flexible and reconfigurable radios (signal waveforms, hardware and transmission techniques) is a key for success as it can enable agile and efficient service multiplexing. [3], [4]

One of the most established technology towards this trend is the beamsteering functionality, [5] which has proven capabilities to provide higher data rates, as well as improved positioning accuracies. On the other hand, to achieve full capabilities of proposed future communication systems, the requirement to adjust all aforementioned radio reconfiguration parameters is clear.

Furthermore, direct corollary of very different requirements, risen by variety of different applications, is the trade-offs between performance metrics. Then, each performance metric is a function of a different set of radio parameters. Thus a joint analysis is a requisite to have a clear insight into the overall performance of the communication system.

The remainder of this article is organized as follows. First, we present the $\mathrm{mmW}$ single-user multiple-input-multipleoutput (MIMO) communication system. We continue by introducing the radio reconfiguration parameters under this study, formulate the performance metrics as well as provide light analysis about the effect of those parameters. We extend the analysis via simulation results. Finally, conclusions are drawn.

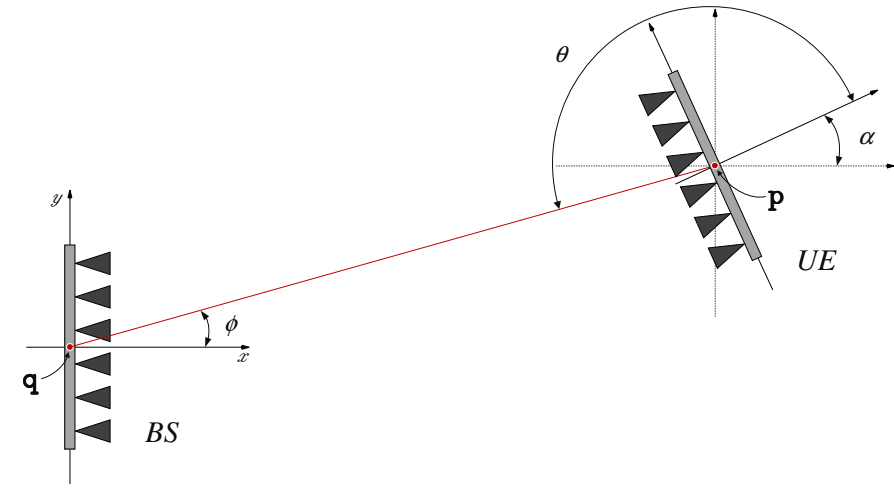

Fig. 1. Communication Model showing transmitter (BS) at location $\mathbf{q}$ and receiver (UE) at location $\mathbf{p}$ with LOS link and the angles: AoA $\phi$, AoD $\theta$, and the rotation $\alpha$ of the receiver.

\section{MMW SINGLE-USER MIMO SYSTEM}

\section{A. Communication Model}

Consider a mmW single-user MIMO wireless system. The location of the transmitter, base-station (BS), and receiver, user equipment (UE), are denoted by $\mathbf{q} \in \mathbb{R}^{2}$ and $\mathbf{p} \in \mathbb{R}^{2}$, respectively. We assume that $\mathbf{q}$ is known, whereas $\mathbf{p}$ is not. As illustrated in Figure 1, we also consider that BS and UE have different orientation with respect to a common reference system. This relative 2D-rotation is hereafter denoted by $\alpha$.

Without loss of generality, we consider that both transmitter and receiver are equipped with uniform linear array (ULA) with $M$ isotropic elements, $0 \mathrm{dBi}$ element gain and $d=\lambda / 2$ element separation, where $\lambda$ is the carrier wavelength.

Due to the "quasi"-optical propagation properties of the mmW channel [2], we assume a single-path MIMO channel $\mathbf{H}(\theta, \phi)$ given by

$$
\mathbf{H}(\theta, \phi)=h \mathbf{a}_{R}(\theta) \mathbf{a}_{T}(\phi)^{\mathrm{H}},
$$

where $h \in \mathbb{C}$ is the dominant channel coefficient, $\mathbf{a}_{T}(\phi), \mathbf{a}_{R}(\theta) \in \mathbb{C}^{M}$ are the transmit and receive array response vectors for the angle-of-departure (AoD) $\phi$ and angleof-arrival (AoA) $\theta$ with

$$
[\mathbf{a}(\theta)]_{m}=e^{j \frac{2 \pi d}{\lambda}(m-1) \sin (\theta)}, m \in\{1, \ldots, M\} .
$$

The received signal is obtained as

$$
y(t)=\sqrt{P_{\mathrm{tx}}} \mathbf{w}^{\mathrm{H}} \mathbf{H}(\theta, \phi) \mathbf{f} x(t-\tau)+\mathbf{w}^{\mathrm{H}} \mathbf{n}(t),
$$


where $\quad x(t)$ is the transmitted signal with $1 / T_{\text {sym }} \int_{0}^{T_{\text {sym }}}|x(t)|^{2} \mathrm{~d} t=1, T_{\text {sym }}$ is the signal duration, $\mathbf{f} \in \mathbb{C}^{M}$ and $\mathbf{w} \in \mathbb{C}^{M}$ are the transmit and receive unit-norm beamforming vectors, generated from codebooks $\mathcal{F}$ and $\mathcal{W}$ with size $N_{t}^{b}=N_{r}^{b}=N^{b}$, respectively; $\mathbf{n}(t)$ is Additive White Gaussian Noise (AWGN) with power spectral density (PSD) $N_{0}, \tau=\|\mathbf{q}-\mathbf{p}\| / c$ is the time-delay of the LOS path for speed of light $c$ and $P_{\mathrm{tx}}$ is the transmission power. Note that $\phi$ and $\theta$ are related through $\alpha=\pi-\theta+\phi$.

Communication occurs over frames of duration $T_{r}=$ $n T_{\text {sym }}$, of which a time $T_{t}=q T_{\text {sym }}$ is devoted to initial access or beamtraining (i.e., determining the best $\mathbf{w}$ and $\mathbf{f}$ ), with $q \leq n, n, q \in \mathbb{N}_{+}$, while the remainder $T_{d}=T_{r}-T_{t}$ is used for data transmission.

\section{B. Initial access}

Prior data communication, BS and UE perform the initial access procedure, which has the objectives to detect the presence of the BS, associate the UE and determine suitable beamforming directions on which subsequent directional communication can be carried out.

In the 3GPP, two procedures have been proposed for the initial access, namely, exhaustive and hierarchical (multi-stage) beam sweeping [6].

In the exhaustive method both transmit and receiver sweep all beams of the codebook seeking for the pair yielding the maximum Signal-to-Noise Ratio (SNR). If $\mathcal{F}$ and $\mathcal{W}$ are orthogonal codebooks, for instance, with $\mathbf{f}_{i}$ and $\mathbf{w}_{j}$ as the $i$-th and $j$-th column of a Discrete Fourier Transform (DFT) $M$ size matrix, respectively, then the total training time (scanning and feedback) for exhaustive strategy is $T_{t}^{e}=T_{s}^{e}+T_{f}^{e}$, where

$$
\begin{aligned}
& T_{s}^{e}=T_{\text {sym }} M^{2}, \\
& T_{f}^{e}=T_{\text {sym }} .
\end{aligned}
$$

In the hierarchical strategy both BS and UE start by partitioning the whole angular domain in $K$ bins and design a beamformer for each bin. Next, they proceed with an exhaustive search mechanism using the $K$ beamformers to select the beam-pair yielding the maximum SNR. Following, they iterate partitioning and search procedures until the narrowest beams are used of an hierarchical codebook are used [7]. It is easy to verify that the total training time (scanning and feedback) for hierarchical strategy is $T_{t}^{h}=T_{s}^{h}+T_{f}^{h}$, with

$$
\begin{aligned}
& T_{s}^{h}=T_{\text {sym }} K^{2}\left(\left\lceil\log _{K}(M)\right\rceil-1\right), \\
& T_{f}^{h}=T_{\text {sym }}\left(\left\lceil\log _{K}(M)\right\rceil-1\right) .
\end{aligned}
$$

\section{Performance metrics}

We consider two performance metrics: effective data rate $R$ and position-rotation error bound (PREB).

- effective data rate: Assuming the beams selected for data transmission, after beam alignment, are $\mathbf{w}$ and $\mathbf{f}$, then

$$
R=B\left(1-\frac{T_{t}}{T_{r}}\right) \log _{2}\left(1+\frac{|h|^{2} P_{\mathrm{tx}} S(\mathbf{w}, \mathbf{f}, \theta, \phi)}{\sigma^{2}}\right),
$$

where $S(\mathbf{w}, \mathbf{f}, \theta, \phi) \triangleq\left|\mathbf{w}^{H} \mathbf{a}_{R}(\theta)\right|^{2}\left|\mathbf{f}^{H} \mathbf{a}_{T}(\phi)\right|^{2}, \sigma^{2}=$ $N_{0} B$ is the noise power over the signal bandwidth $B$. Note that for a fixed $T_{t}$, the rate is maximized when $\mathbf{f}=1 / \sqrt{M} \mathbf{a}_{T}(\phi)$ and $\mathbf{w}=1 / \sqrt{M} \mathbf{a}_{R}(\theta)$.

- position-rotation bound: Assuming the initial access procedure is used for positioning [8], [9], then the achievable Position Error Bound (PEB) and Rotation Error Bound (REB) are computed as

$$
\begin{aligned}
& \mathrm{PEB}=\sqrt{\sigma_{x}^{2}+\sigma_{y}^{2}}, \\
& \mathrm{REB}=\sqrt{\sigma_{\alpha}^{2}},
\end{aligned}
$$

where $\sigma_{x}^{2}, \sigma_{y}^{2}$ and $\sigma_{\alpha}^{2}$ are obtained from the inverse of the Fisher Information Matrix (FIM) associated with the $\mathrm{mmW}$ based positioning problem [8], [9].

\section{5G RADIO RECONFIGURATION AND IMPACT ON DATA RATE AND POSITIONING}

Hereafter, we consider a reconfigurable $5 \mathrm{G}$ radio where, for instance, signal and antenna radiation patterns are reconfigurable by RadioApps [10]. More specifically, we focus on orthogonal frequency-division multiplexing (OFDM) waveform reconfiguration [11] and electronically reconfigurable arrays as they are potential candidates for future 5G systems [12].

\section{A. Waveform reconfiguration}

To begin with, let us investigate the impact of OFDM subcarrier spacing [11], [13]-[15] on the initial access overhead, thus on the effective rate. As shown in equation (11), for a given $T_{r}$, number of antenna elements $M$, initial access strategy and codebooks, the $R$ depends on inter-carrier spacing $\Delta_{f}$ as follows

$$
\begin{aligned}
R\left(\Delta_{f}\right)= & B\left(\Delta_{f}\right)\left(1-\frac{\mathcal{Q}_{t}(M)}{\mathcal{Q}_{r}\left(\Delta_{f}\right)}\right) . \\
& \log _{2}\left(1+\rho\left(\Delta_{\mathrm{f}}, \mathbf{w}^{*}, \mathbf{f}^{*}, \theta, \phi, \mathrm{h}\right)\right),
\end{aligned}
$$

where $\mathcal{Q}_{r}\left(\Delta_{f}\right)$ is the function giving the number of slots in the frame duration, i.e., $T_{r}=\mathcal{Q}_{r}\left(\Delta_{f}\right) T_{\text {sym }}, \mathcal{Q}_{t}(M)$ is the function providing the number of slots necessary for the initial access given the number of antennas, i.e., $T_{t}=\mathcal{Q}_{t}(M) T_{\text {sym }}$, and $\rho\left(\Delta_{f}, \mathbf{w}^{*}, \mathbf{f}^{*}, \phi, \theta, h\right)$ is the SNR achieved after beam alignment with $\mathbf{w}^{*}, \mathbf{f}^{*}$ given by

$$
\left[\mathbf{w}^{*}, \mathbf{f}^{*}\right]=\arg \max _{\substack{\mathbf{w} \in \mathcal{W} \\ \mathbf{f} \in \mathcal{F}}} \frac{|h|^{2} P_{\mathrm{tx}} \mathrm{S}(\mathbf{w}, \mathbf{f}, \theta, \phi)}{N_{0} B\left(\Delta_{f}\right)},
$$

where $B\left(\Delta_{f}\right)$ is the signal bandwidth that is function of $\Delta_{f}$ and the number of subcarrier used.

From the above, we notice that the impact of $\Delta_{f}$ on the effective rate is three-fold. First, larger the subcarrier spacing, the higher the signal bandwidth is. Second, larger the subcarrier spacing, the lower the overhead is. Subsequently, the rate increases both because $B$ and the number of slots for data communication are larger. Third, we notice that the logarithmic term decreases with $\Delta_{f}$ growing. Therefore, a tension between the decrease of the SNR and the increase of the bandwidth as well as the number of slots is expected. 
Next, we analyse the impact of $\Delta_{f}$ on the PREB. In [8], it is shown that the PEB and REB can be computed from the inverse of the FIM $\mathbf{J}_{\xi}$ associated with the estimation of $\xi \triangleq[\mathbf{p}, \alpha, h]^{\mathrm{T}}$. More specifically, the FIM $\mathbf{J}_{\boldsymbol{\xi}}$ is computed as

$$
\mathbf{J}_{\boldsymbol{\xi}}=\sum_{\substack{\mathbf{w} \in \mathcal{W} \\ \mathbf{f} \in \mathcal{F}}} \mathbf{J}_{\boldsymbol{\xi}}(\mathbf{f}, \mathbf{w})
$$

where the FIM associated with a single beam pair is denoted by $\mathbf{J}_{\boldsymbol{\xi}}(\mathbf{f}, \mathbf{w})$ and obtained with the bilinear transformation

$$
\mathbf{J}_{\boldsymbol{\xi}}(\mathbf{f}, \mathbf{w})=\mathbf{T} \mathbf{J}_{\boldsymbol{\eta}}(\mathbf{f}, \mathbf{w}) \mathbf{T}^{\mathrm{T}}
$$

where $\mathbf{J}_{\boldsymbol{\eta}}(\mathbf{f}, \mathbf{w})$ is the FIM associated with the estimation of $\boldsymbol{\eta} \triangleq[\tau, \phi, \theta, h]^{\mathrm{T}}$ and $\mathbf{T} \in \mathbb{R}^{5 \times 5}$ is

$$
\mathbf{T}=\left[\begin{array}{cc}
\mathbf{\Upsilon} & \mathbf{0} \\
\mathbf{0} & \mathbf{I}_{2}
\end{array}\right],
$$

with $\mathbf{I}_{2}$ is $2 \times 2$ identity matrix and $\boldsymbol{\Upsilon}$ given by

$$
\mathbf{\Upsilon}=\frac{1}{c}\left[\begin{array}{ccc}
\cos (\phi) & -\sin (\phi) / \tau & -\sin (\phi) / \tau \\
\sin (\phi) & \cos (\phi) / \tau & \cos (\phi) / \tau \\
0 & 0 & -c
\end{array}\right],
$$

where $c$ indicates the speed-of-light.

In [8], it is also shown that all non-zero terms in $\mathbf{J}_{\boldsymbol{\eta}}$ are proportional to $E_{s} / N_{0}$, thus to $P_{\mathrm{tx}} /\left(B\left(\Delta_{f}\right) N_{0}\right)$. Moreover, $J_{\tau, \tau}$ (ranging information term) is also proportional to $B\left(\Delta_{f}\right)^{2}$. In light of the above the following observations are in order.

On the one hand, the larger $\Delta_{f}$, the lower the ranging error is. On the other hand, the larger $\Delta_{f}$, the worse AoD and AoA estimation are since $E_{s}$ decreases. Also, the reduction of $E_{s}$ affects the cross-terms in $\mathbf{J}_{\boldsymbol{\eta}}$, which, in turn, have an impact on the $\mathbf{J}_{\boldsymbol{\xi}}$ due to the bilinear transformation in (14). Finally, due to the summation in (13), the choice of initial access strategy and choice of the codebook have an impact too.

Unlike the rate, the impact of $\Delta_{f}$ on the PEB and REB is not straightforward and, in the next Section, we will investigate this connection via simulations and try to gain useful insights.

\section{$B$. Antenna reconfiguration}

We consider the possibility to digitally control the on-off state of elements of an antenna array to either decrease (or increase) the beamwidth, thus increase (or decrease) the array gain. In [8], [16], the impact on the effective rate and PEB due to the initial access overhead is studied in details. Generally, it was shown that an optimal beamwidth size can be determined for the exhaustive beam sweeping strategy. Also, that the optimal beamwidth size depends on the distance as well as the duration of $T_{r}$. On the other hand, for the hierarchical beam sweep strategy, there is not a trade-off to be met.

Therefore, in this paper, we simply utilize the antenna reconfigurability as a tool to change the beams for the initial access, thus the number of training slots i.e. $\mathcal{Q}(M)$.

\section{Simulations Results}

In this Section we study the effect of the radio parameters, namely subcarrier spacing $\Delta_{f}$, number of active antennas, for achievable data rate and position-rotation error bound as well as the trade-off between these two metrics. Different initial access strategies are considered, too.

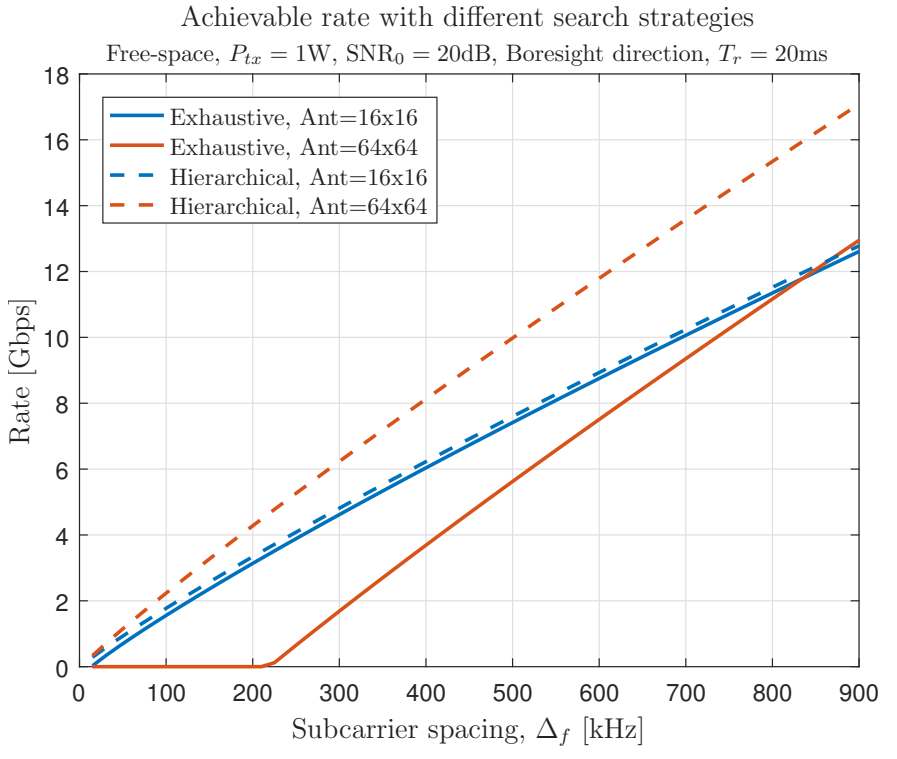

Fig. 2. Achievable rate as a function of subcarrier spacing $\Delta_{f}$ and number of antennas $M$.

\section{A. Simulation setup}

The system in our investigation is an idealized MIMOOFDM communication system with 2048 subcarriers, fixed transmission power $P_{t x}=30 \mathrm{dBm}$, carrier frequency $28 \mathrm{GHz}$, and equal number of antenna elements both on the transmitter and receiver. During the initial access, one beamformer is used to transmit one OFDM symbol with energy $E_{s}=P_{t x} T_{s y m}$.

The frame duration (initial access and data transmission) is fixed and it is equal to $20 \mathrm{~ms}$. However, based on $\Delta_{f}$, thus $T_{\text {sym }}$, the total number of OFDM slots per frame varies.

The scenario consists an UE located a distance of $100 \mathrm{~m}$ from the $\mathrm{BS}$, corresponding $\mathrm{SNR}_{0} \approx 20 \mathrm{~dB}$, where $\mathrm{SNR}_{0} \triangleq$ $\mathrm{dB}\left(|h|^{2} E_{s} / N_{0}\right)$. Furthermore, UE is assumed to face $\mathrm{BS}$ perfectly, i.e. antenna boresight.

\section{B. Trade-off between rate and subcarrier spacing}

The first result is illustrated in Figure 2 showing the achievable rate as a function of subcarrier spacing $\Delta_{f}$ for two different configurations of active antenna elements, $M=16$ and $M=64$, and for two different beamsearch strategies, exhaustive search and hierarchical search.

First, let us look at the performance of the exhaustive search (solid lines), and compare the case with $M=16$ to the one with $M=64$ (blue and red lines, respectively). It can be noticed that with $M=16$ the achievable data rate grows nearly linearly as function of $\Delta_{f}$. Then, in case $M=64$ exhaustive search strategy requires at minimum $\Delta_{f}>235 \mathrm{kHz}$ to have training time $T_{r}^{e}<T_{t}$, consequently having data rate $>0$. Clearly, this is directly related to the length of $T_{r}$ and the codebook (DFT-based int this case).

Next, we observe that with $M=16$, the data is higher than that with case $M=64$ until the crossing point $\Delta_{f} \approx 850 \mathrm{kHz}$. This is explained by analysing equation (11) as a function of $\Delta_{f}$ given $M$. More specifically, in [16] it is shown that 


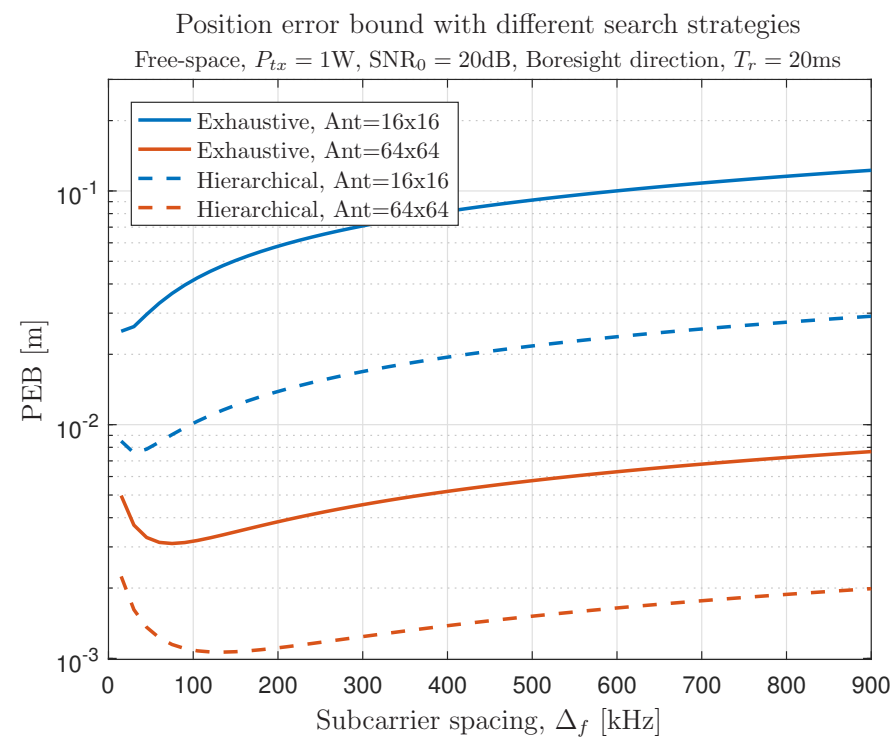

Fig. 3. Position error bound as a function of subcarrier spacing $\Delta_{f}$ and number of antennas $N=M$.

$\mathcal{Q}_{t}(M)=M^{2}$, thus $\left(1-M^{2} / \mathcal{Q}_{r}\left(\Delta_{f}\right)\right)$ decreases faster with $M$ larger. Furthermore, the difference in slopes results that the selection of the optimal number of active antennas depends on subcarrier spacing.

Second, let us look the hierarchical search (dashed lines), and compare again the case with $M=16$ to the $M=64$. It can be noticed that in both cases the achievable data rate is not reduced to zero even with small subcarrier spacing.In other words, the number of slots even with high number of antennas $M=64$ and a small subcarrier spacing $\Delta_{f}=15 \mathrm{kHz}$ is enough to cover the initial access time within the hierarchical search procedure. Then again, the greater value of $N$ results the more steep performance curve measuring achievable data rate. In contrast with the exhaustive search, the case $M=64$ in the hierarchical search results better performance in data rate with all $\Delta_{f}>15 \mathrm{kHz}$.

\section{Trade-off between position-rotation error bound and sub- carrier spacing}

In this section we study the position-rotation error bound as function of $\Delta_{f}$ as well as a selection of number of active antennas and different search strategies.

Figure 3 shows the PEB as a function of subcarrier spacing $\Delta_{f}$, for two different antenna settings as well as beamsearch strategies. Our first observation is that the selection of beamsearch strategy as well as selection of number of active antennas provides direct gain in performance of PEB. Furthermore, the general trend is that PEB grows as a function of $\Delta_{f}$.

Then, we observe that the PEB is affected not only by $\Delta_{f}$, but also SNR, the choice of initial access strategy and choice of the codebook, underlining the discussion in Section III-A. The out-turn is that the optimal performance in PEB as function of $\Delta_{f}$ is also affected by aforementioned factors. This can

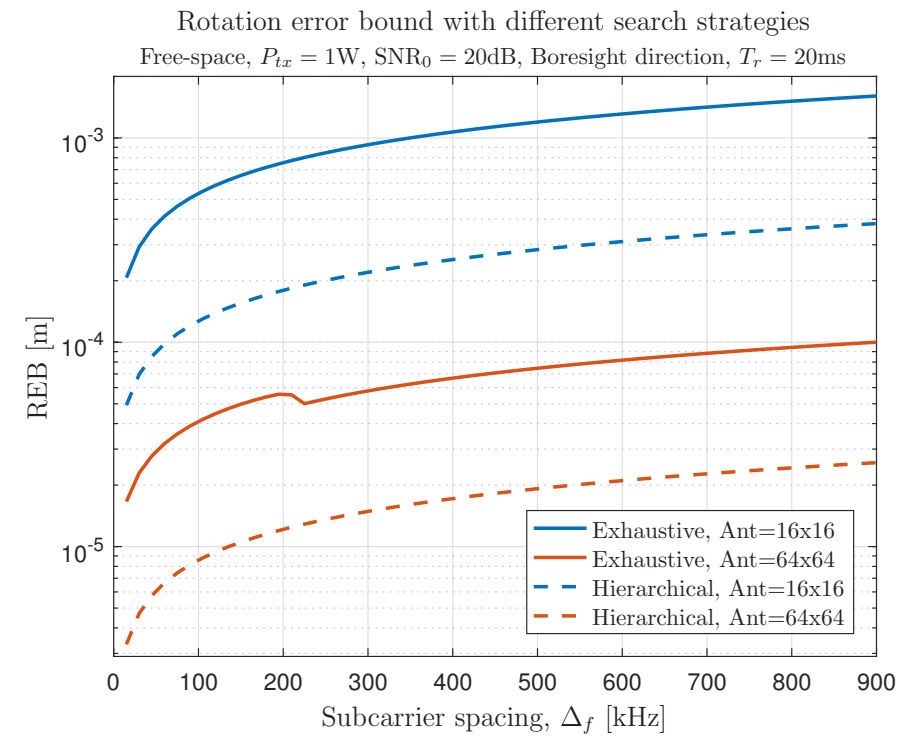

Fig. 4. Rotation error bound as a function of subcarrier spacing $\Delta_{f}$ and number of antennas $N=M$.

be seen in Figure 3, e.g. by looking the case of hierarchical search and while with $M=16$ the minimum point of PEB is $\Delta_{f} \approx 30 \mathrm{kHz}$, with $M=64$ it is $\Delta_{f} \approx 120 \mathrm{kHz}$. Additionally, a similar phenomenon also exists in case with $M=64$ and comparing the exhaustive search with the hierarchical search.

Next we study the results depicted in Figure 4 which shows REB in same aforementioned scenarios. First we note the similar performance as within the performance of PEB, namely REB is clearly affected by the selection of beamsearch strategy as well as by the number of active antennas. Then, each case shown in Figure 4 is degreasing as a function of $\Delta_{f}$.

Next, we observe a notch in exhaustive search case with $M=64$. This is reflected to the behaviour of the achievable rate with $M=64$, as the rate $=0$ until the $\Delta_{f}$ is large enough to provide enough slots to carry the full beamsearch loop.

Until the aforementioned cutting point is reached, there exists an imbalance between the AoA and AoD estimation, namely a difference between the search directions carried out within the search loop. This imbalance is cleared up when the cutting point is reached by $\Delta_{f}$. Furthermore, as the notch is due to the number of slots, it is clear that the location of the notch is also function of frame length $T_{r}$.

\section{Trade-off between rate and position-rotation bound}

To conclude analysis of simulation results, we review the performance of the system in achievable data rate also as a function of PEB and REB.

First, results shown in Figure 5 underline the discussion in Section III-A. Particularly, in all cases achievable data rate grows as function of $\Delta_{f}$ and also all cases achieves distinctly different performance rates.

Secondly, results presented in Figure 5 shows that REB grows as a function of $\Delta_{f}$ as well as give a clear indication about the trade-off between achievable data rate and the rotation estimation. 


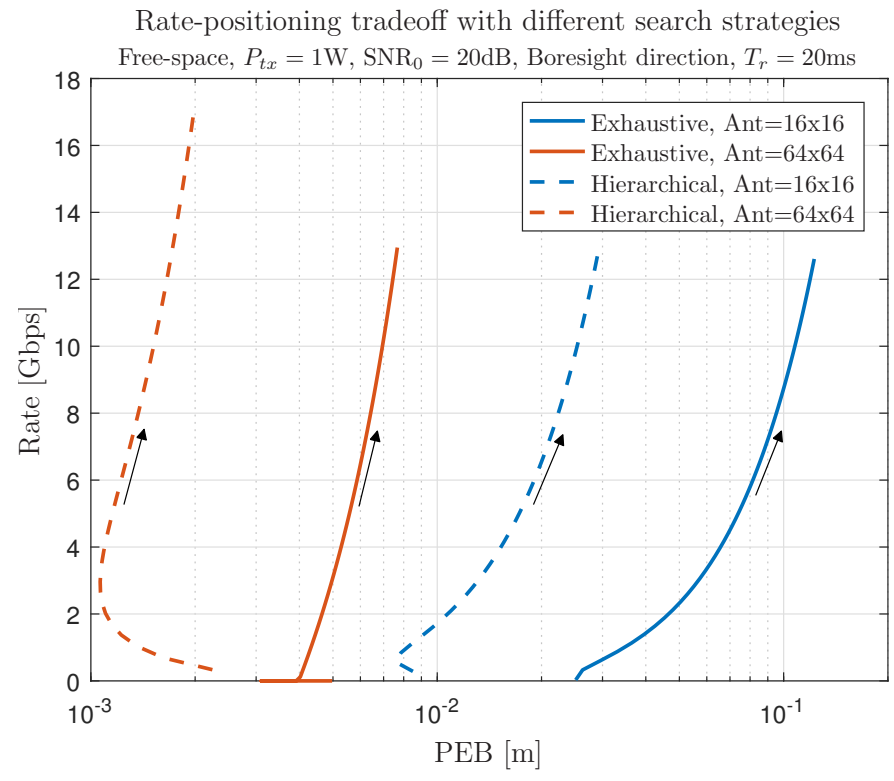

Fig. 5. Rate as function of position error bound and number of antennas $N=M$. The black arrow indicating the direction in which subcarrier spacing $\Delta_{f}$ is growing.

\section{CONCLUSiON}

In this paper we considered a mmW-MIMO communication system and investigated the effect of configurable parameters to the achievable data rate and position capabilities. The following conclusions were drawn: (i) to achieve best possible data rate, a selection of number of active antennas depends on subcarrier spacing with the exhaustive beamsearch strategy oppositely with the hierarchical search strategy the selection of number of active antennas does not depend on subcarrier spacing; (ii) common trend for positioning performance, as shown for PEB as well as for REB, is that smaller the subcarrier spacing $\Delta_{f}$, better the performance; and (iii) a tradeoff between data rate and achievable positioning accuracy provides an application specific recourse to meet different requirements.

\section{ACKNOWLEDGMENTS}

The research leading to these results has received funding from the Academy of Finland, Fundamental of Simultaneous Localization and Communications (FALCON) as well as the European Union H2020 5GPPP under grant n. 723247 and supported by the Institute for Information \& communications Technology Promotion (IITP) grant funded by the Korea government (MSIP) (No.B0115-16-0001, 5GCHAMPION).

\section{REFERENCES}

[1] M. Mueck et al., "5G CHAMPION - Rolling out 5G in 2018," in Proc. IEEE Global Commun. Conf. Workshops, Dec. 2016, pp. 1-6.

[2] T. S. Rappaport, S. Sun, R. Mayzus, H. Zhao, Y. Azar, K. Wang, G. N. Wong, J. K. Schulz, M. Samimi, and F. Gutierrez, "Millimeter Wave Mobile Communications for 5G Cellular: It Will Work!" vol. 1, pp. 335-349, 2013.

[3] J. G. Andrews, S. Buzzi, W. Choi, S. V. Hanly, A. Lozano, A. C. K. Soong, and J. C. Zhang, "What Will 5G Be?" vol. 32, no. 6, pp. 10651082, Jun. 2014.

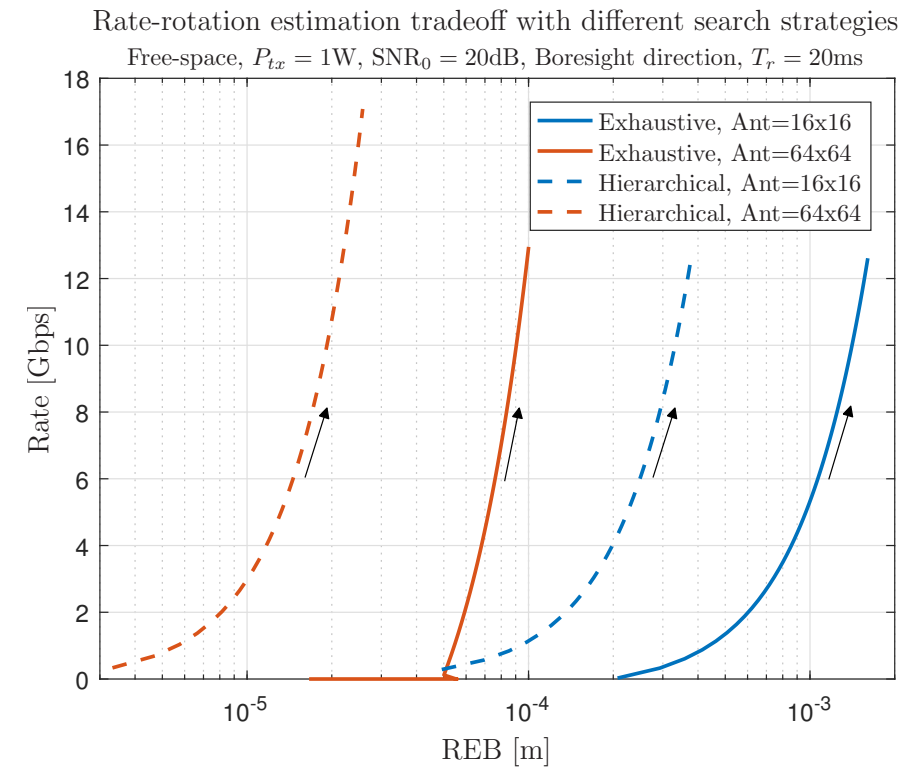

Fig. 6. Rate as function of rotation error bound and number of antennas $N=M$. The black arrow indicating the direction in which subcarrier spacing $\Delta_{f}$ is growing.

[4] R. W. Heath, N. González-Prelcic, S. Rangan, W. Roh, and A. M. Sayeed, "An Overview of Signal Processing Techniques for Millimeter Wave MIMO Systems," IEEE J. Sel. Topics Signal Process., vol. 10, no. 3, pp. 436-453, Apr. 2016.

[5] S. Kutty and D. Sen, "Beamforming for Millimeter Wave Communications: An Inclusive Survey," IEEE Commun. Surveys Tuts., vol. 18, no. 2, pp. 949-973, 2016.

[6] 3GPP TSG RAN, "R1-167115 - Discussion on Beam Sweeping for Initial Access," 3GPP TSG RAN WG1 Meeting \#86, Aug. 2016.

[7] Z. Xiao, T. He, P. Xia, and X. G. Xia, "Hierarchical codebook design for beamforming training in millimeter-wave communication," IEEE Trans. Wireless Commun., vol. 15, no. 5, pp. 3380-3392, May 2016.

[8] G. Destino and H. Wymeersch, "On the Trade-off Between Positioning and Data-Rate for mm-Wave Communications," in Proc. IEEE Int. Conf. Commun. Workshop, May 2017.

[9] A. Shahmansoori, G. E. Garcia, G. Destino, G. Seco-Granados, and H. Wymeersch, "Position and Orientation Estimation through Millimeter Wave MIMO in 5G Systems," ArXiv e-prints, Feb. 2017.

[10] M. Mueck, V. Ivanov, S. Choi, J. Kim, C. Ahn, H. Yang, G. Baldini, and A. Piipponen, "Future of wireless communication: Radioapps and related security and radio computer framework," IEEE Wireless Commun. Mag., vol. 19, no. 4, pp. 9-16, Aug. 2012.

[11] A. A. Zaidi, R. Baldemair, H. Tullberg, H. Bjorkegren, L. Sundstrom, J. Medbo, C. Kilinc, and I. D. Silva, "Waveform and Numerology to Support 5G Services and Requirements," IEEE Commun. Mag., vol. 54, no. 11, pp. 90-98, Nov. 2016.

[12] L. D. Palma, A. Clemente, L. Dussopt, R. Sauleau, P. Potier, and P. Pouliguen, "Circularly-Polarized Reconfigurable Transmitarray in KaBand With Beam Scanning and Polarization Switching Capabilities," IEEE Trans. Antennas Propag., vol. 65, no. 2, pp. 529-540, Feb. 2017.

[13] E. Lähetkangas, K. Pajukoski, J. Vihriälä, G. Berardinelli, M. Lauridsen, E. Tiirola, and P. Mogensen, "Achieving low latency and energy consumption by 5G TDD mode optimization," in Proc. IEEE Int. Conf. Commun. Workshop, Jun. 2014, pp. 1-6.

[14] F. Schaich, T. Wild, and R. Ahmed, "Subcarrier Spacing - How to Make Use of This Degree of Freedom," in Proc. IEEE Veh. Technol. Conf. (Spring), May 2016, pp. 1-6.

[15] 3GPP TSG RAN, "R1-162386 - Numerology for new radio interface," 3GPP TSG RAN WG1 Meeting \#84bis, Apr. 2016.

[16] J. Saloranta, G. Destino, and H. Wymeersch, "Comparison of Different Beamtraining Strategies from a Rate-Positioning Trade-Off Perspective," in Proc. Eur. Conf. Networks and Commun., 2017. 